Article

\title{
Effect of Selenium Enrichment and Type of Application on Yield, Functional Quality and Mineral Composition of Curly Endive Grown in a Hydroponic System
}

\author{
Leo Sabatino ${ }^{1}\left(\mathbb{D}\right.$, Georgia Ntatsi ${ }^{2,3}{ }^{\circledR}$, Giovanni Iapichino ${ }^{1, *}$, Fabio $D^{\prime}$ Anna ${ }^{1}$ and \\ Claudio De Pasquale ${ }^{1}$ (D) \\ 1 Dipartimento Scienze Agrarie, Alimentari e Forestali, Università di Palermo, Viale delle Scienze, \\ 90128 Palermo, Italy; leo.sabatino@unipa.it (L.S.); fabio.danna@unipa.it (F.D.); \\ claudio.depasquale@unipa.it (C.D.P.) \\ 2 Laboratory of Vegetable Production, Department of Crop Science, Agricultural University of Athens, \\ 11855 Athens, Greece; ntatsi@aua.gr \\ 3 Institute of Plant Breeding and Genetic Resources, Hellenic Agricultural Organization-ELGO DEMETER, \\ 57001 Thessaloniki, Greece \\ * Correspondence: giovanni.iapichino@unipa.it; Tel.: +39-091-23862215
}

Received: 16 March 2019; Accepted: 22 April 2019; Published: 24 April 2019

check for updates

\begin{abstract}
Selenium (Se) is an essential element for humans' health and the recommended amount (RDA) of Se intake per adult is 55-70 $\mu \mathrm{g} \mathrm{day}^{-1}$. The main source for Se intake is represented by diet, but its concentration in vegetables is generally limited with respect to human needs. The aim of this research was to assess the effect of Se application rate and type (fertigation or foliar spray) on yield, functional properties and mineral composition of curly endive grown in a hydroponic system. Five levels of Se $\left(0.0,1.0,2.0,4.0\right.$ and $\left.8.0 \mu \mathrm{mol} \mathrm{L}^{-1}\right)$ were supplied via fertigation or by foliar spray

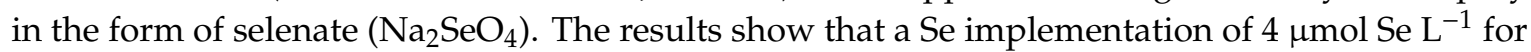
plants enriched via fertigation and $8 \mu \mathrm{mol} \mathrm{Se} \mathrm{L}^{-1}$ for plants biofortified via foliar spray successfully enhanced production performance parameters such as head weight ( $42.6 \%$ and $27.8 \%$, respectively), SSC $(16.7 \%$ and $14.3 \%$, respectively), ascorbic acid (33.2\% and $33.7 \%$, respectively), total phenolic (58.9\% and $54.5 \%$, respectively) and Se concentration (22.2\% and $20.3 \%$, respectively). Furthermore, leaf Se concentration in plants enriched via fertigation ranged $0.71-17.61 \mathrm{mg} \mathrm{kg}^{-1}$ of dry weight (DW), whereas, in plant biofortified via foliar spray leaf Se concentration ranged $0.72-12.67 \mathrm{mg} \mathrm{kg}^{-1}$ DW. Plants grown with the highest dosage of Se distributed via fertigation or foliar spray showed a reduction in total-N leaf concentration by $39.4 \%$ and $28.6 \%$, respectively, compared with the non-enriched plants. Our results indicate a consumption of $47.4 \mathrm{~g}$ day $^{-1}$ of Se-enriched curly endive grown in soilless culture and treated with $8 \mu \mathrm{mol} \mathrm{L}^{-1}$ of selenate applied via foliar spray could be sufficient to cover the human physiological needs of this element.
\end{abstract}

Keywords: Cichorium endivia; biofortification; soilless culture; nutrition; ascorbic acid

\section{Introduction}

Humans need different elements for a good health and development, which can be supplied by an adequate diet. However, the world's population suffers of mineral deficiency including Selenium (Se) shortage (about $15 \%$ of the world's population are Se deficient) [1]. Mineral malnourishment can be overcome through well-chosen dietary diversification, mineral supplementation, food fortification and by increasing the bio-available mineral concentration in edible crops (a process called 
biofortification) [2-4]. Selenium has been considered essential to animal and human nutrition since 1957 as a component of the enzymes glutathione peroxidase, selenoprotein $\mathrm{P}$, and tetraiodothyronine $5^{\prime}$-deiodinase [5]. Selenium is an element that determines the normal functioning of an organism [6], but unlike all other elements, selenium has one of the narrowest ranges between dietary deficiency $\left(<40 \mu \mathrm{g} \mathrm{day}^{-1}\right)$ and toxic levels $\left(>400 \mu \mathrm{g} \mathrm{day}^{-1}\right)$. Gissel-Nielsen et al. [7] and Marschner [8] reported

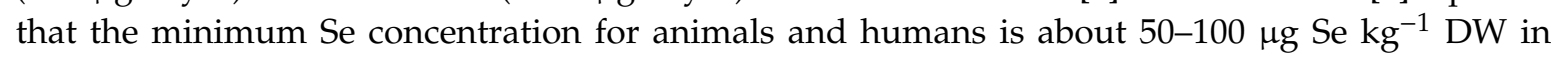
fodder/food. According to U.S. Department of Agriculture [5], the humans' daily requirement is 50-70 $\mu \mathrm{g}$. Low Se dietary intake can be associated with health disorders such as oxidative stress-related conditions, hypothyroidism, weakened immune system, cardiovascular disease, reduced male fertility, and increased risk of cancer [9-11]. Conversely, adequate Se consumption through human diet has health benefits besides meeting basic nutritional needs. For instance, some organic forms of Se such as methyl-selenocysteine (MSeC) have been reported to exhibit anticarcinogenic activity against different types of cancer [12]. Recent studies demonstrated the role of plants as the main dietary source of this element; consequently, there has been an increasing effort to augment the Se content in plants used for human consumption [13-17]. In this respect, the introduction of this element through fertigation represents a very effective way to overcome Se low bioavailability.

Curly endive (Cichorium endivia L. var. crispum Hegi) is appreciated for its distinctive crunchy texture and mildly bitter taste, making it suitable for direct consumption as well as an ingredient of mixed ready-to-eat salads. Furthermore, curly endive contains high levels of ascorbic acid and minerals such as potassium and calcium [18]. However, selenium concentration in curly endive and in other vegetable crops as well, is generally lower than $1.0 \mathrm{mg} \mathrm{kg}^{-1}$ dry weight $[1,13,14,16,17]$. Hence, increasing the Se concentration in leaves of curly endive via fertilization could be beneficial to human health. Hydroponics is the usual growth system for curly endive; however, open field and conventional greenhouse cultivation is common in Mediterranean climates. Fertigation and foliar application are simple, efficient and practical methods for plant nutrient supply. However, since foliar and root absorption are affected by genotype and growing conditions, specific studies are required to assess methods and doses. To the best of our knowledge, the literature lacks information on the interaction between Se fertilization dosage and application form in curly endive and their effects on its quantitative and qualitative traits. Taking all the above into consideration, an experiment was conducted to assess the effect of Se application rate and type (fertigation or foliar spray) on yield, functional properties (phenolics and ascorbic acid) and mineral composition of curly endive grown in a hydroponic system.

\section{Materials and Methods}

\subsection{Plant Material and Growing Conditions}

The experiment was conducted under greenhouse conditions at the experimental field of the Department of Agricultural, Food and Forest Sciences of Palermo (SAAF), at Marsala, Trapani Province (longitude $12^{\circ} 26^{\prime} \mathrm{E}$, latitude $37^{\circ} 47^{\prime} \mathrm{N}$, altitude $37 \mathrm{~m}$ ) on the northwestern coast of Sicily (Italy). The high-tech greenhouse was equipped with a fan-and-pad evaporative cooling, high-pressure fogging and over-head air heating systems. On 1 March 2017, seedlings at the stage of four to five true leaves of curly endive (Cichorium endivia L., var. crispum Hegi) (var. Salad King, Topseed s.r.l., Sarno, Italy) were transplanted in drilled polystyrene panels $\left(0.5 \mathrm{~m} \times 1.0 \mathrm{~m} ; 12\right.$ plants $\left.\mathrm{m}^{2}\right)$.

Curly endive plants were grown in an open hydroponic floating system using nutrient solutions, with five levels of selenium $\left(0,1.0,2.0,4.0\right.$ and $\left.8.0 \mu \mathrm{mol} \mathrm{L} \mathrm{L}^{-1}\right)$ distributed via fertigation or foliar spray. The four foliar treatments were conducted every two weeks starting on 15 March and finishing on 26 April. For every foliar spray application, the volume used was $1.5 \mathrm{~L} \mathrm{~m}^{-2}$. The different Se levels were attained by adding appropriate amounts of selenate- $-\mathrm{Na}_{2} \mathrm{SeO}_{4}$ (Sigma-Aldrich, Saint Louis,

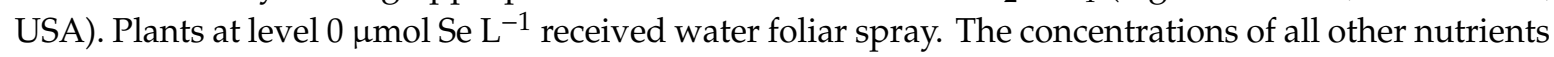
in the solution initially introduced into the system were identical for all nutrient solution levels and the composition was as follows: $4.50 \mathrm{mmol} \mathrm{L}^{-1}$ of $\mathrm{Ca}^{2+}, 2.00 \mathrm{mmol} \mathrm{L}^{-1}$ of $\mathrm{H}_{2} \mathrm{PO}_{4}^{-}, 1.25 \mathrm{mmol} \mathrm{L}^{-1} \mathrm{NH}_{4}^{+}$, 
$1.00 \mathrm{mmol} \mathrm{L}^{-1}$ of $\mathrm{Mg}^{2+}, 19.00 \mathrm{mmol} \mathrm{L}^{-1}$ of $\mathrm{NO}_{3}{ }^{-}, 11.00 \mathrm{mmol} \mathrm{L}^{-1}$ of $\mathrm{K}^{+}, 1.10 \mathrm{mmol} \mathrm{L}^{-1}$ of $\mathrm{SO}_{4}{ }^{2-}$, $40.00 \mu \mathrm{mol} \mathrm{L}-1$ of Fe, $5.00 \mu \mathrm{mol} \mathrm{L}^{-1}$ of Mn, $4.00 \mu \mathrm{mol} \mathrm{L}{ }^{-1}$ of $\mathrm{Zn} 30.00 \mu \mathrm{mol} \mathrm{L}^{-1}$ of B, and $0.75 \mu \mathrm{mol} \mathrm{L}^{-1}$ of $\mathrm{Cu}$ [19]. The electrical conductivity (EC) and pH in the above nutrient solution were $2.5 \mathrm{mS} \mathrm{cm}^{-1}$ and 5.8, respectively. Each nutrient solution was poured into a rectangular tank $(200 \mathrm{~cm}$ long $\times 100 \mathrm{~cm}$ wide $\times 20 \mathrm{~cm}$ deep) containing $300 \mathrm{~L}$ of nutrient solution. The nutrient solutions were not aerated during the growing period, as the fast growth of the leafy vegetables does not need a high oxygen concentration in the nutrient solution [20]. The nutrient solution was monitored weekly for EC and $\mathrm{pH}$. The $\mathrm{pH}$ in the nutrient solution (NS) was adjusted daily by adding appropriate amounts of $\mathrm{HNO}_{3}$. The tanks were refilled with new nutrient solution when the volume of the NS dropped by $20 \%$.

The five Se levels were combined with the two types of Se application in a two factorial experimental design rendering ten treatments. Each treatment was replicated four times for each Se concentration and type of Se application (36 tanks). Climate conditions inside the greenhouse were adjusted via computer controller and was set to $12 \pm 1{ }^{\circ} \mathrm{C}$ during the night and $18 \pm 1^{\circ} \mathrm{C}$ during the day. Relative humidity was kept within $60-70 \%$ during the growing season. The cumulated greenhouse global radiation was $473.5 \mathrm{MJ} \mathrm{m}^{-2}$.

\subsection{Yield and Morphological Traits}

Harvest took place 70 days after transplanting. Head fresh weight (HFW), head height $(\mathrm{HH})$, stem diameter (SD) and number of leaves (NL) were measured on all plants of the replicates.

Sampling for the dry matter determination and quality analysis was conducted using 5 plants randomly selected in each replicate. Head dry matter content (HDMC) and root dry matter content (RDMC) were obtained through the dehydration of the sample in a heater at $80^{\circ} \mathrm{C}$ for the first two days and subsequently dried at $105^{\circ} \mathrm{C}$ until constant weight using a thermo-ventilated oven (Memmert, Serie standard, Venice, Italy).

\subsection{Functional Quality Analysis}

Samples for functional quality analysis were collected at harvest. Leaf samples were squeezed, the juice was filtered and soluble solids content (SSC) was measured using a digital refractometer (MTD-045nD, Three-In-161 One Enterprises Co. Ltd. Taiwan). Titratable acidity (TA) was determined by potentiometric titration with $0.1 \mathrm{M} \mathrm{NaOH}$ up to $\mathrm{pH} 8.1$, using $15 \mathrm{~mL}$ of plant extract and expressed as percent malic acid equivalents. TA was expressed as percentage of malic acid [21].

Ascorbic acid content was measured from the leaf samples by reflectometer Merck RQflex* $10 \mathrm{~m}$ using Reflectoquant Ascorbic Acid Test Strips. One gram of leaf juice was dissolved in distilled water, to a total of $10 \mathrm{~mL}$, and mixed. Then, appropriate test strip was dipped into the sample and inserted into the meter. Results were expressed as $\mathrm{mg}$ of ascorbic acid per $\mathrm{kg}$ fresh weight.

As for total phenolics, leaf samples of $5 \mathrm{~g}$ were extracted using methanol and was assayed quantitatively by A765. Total phenolics content was measured using Folin-Ciocalteu reagent [22]. The results were expressed as $\mathrm{mg}$ of caffeic acid $\mathrm{g}^{-1}$ fresh weight.

\subsection{Mineral Composition}

Samples for mineral composition analysis were collected at harvest. The Kjeldal method was used to determine leaf total Nitrogen $(\mathrm{N})$. In particular, a sample rate was subjected to acid-catalyzed mineralization to turn the organic nitrogen into ammoniacal nitrogen. The ammoniacal nitrogen was then distilled in an alkaline $\mathrm{pH}$. The ammonia formed during this distillation was collected in a boric acid solution and determined through titrimetric dosage.

$\mathrm{Ca}, \mathrm{Mg}$, and $\mathrm{K}$ were determined using atomic absorption spectroscopy (SavantAA, 200 ERRECI, Milan, Italy) following wet mineralization [23]. Phosphorus levels were determined using colorimetry [24]. With regard to $S$ determination, $500 \mathrm{mg}$ of dried tissues were weighed and acid-digested with $4.0 \mathrm{~mL}$ of concentrated $\mathrm{HNO}_{3}+2.0 \mathrm{~mL}$ of concentrated $\mathrm{HClO}_{4}$ (Sigma-Aldrich, Saint Louis, MO, USA) at $120^{\circ} \mathrm{C}$ 
for $1 \mathrm{~h}$ and then at $220{ }^{\circ} \mathrm{C}$ until $\mathrm{HClO}_{4}$ fumes were observed. Total $\mathrm{S}$ contents in the samples were determined using an atomic absorption spectrophotometer (SavantAA, 200 ERRECI, Milan, Italy).

For the determination of the Se concentration, $25 \mathrm{mg}$ of dried leaf sample were digested with $2.5 \mathrm{~mL}$ of concentrated $\mathrm{HNO}_{3}$ and $1 \mathrm{~mL}$ of $\mathrm{H}_{2} \mathrm{O}_{2}$ in an analytical microwave oven. The resulting solution was diluted to $25 \mathrm{~mL}$ with deionized water and the metal concentration determined by ICP-MS (Plasma Quant MS Elite, Jena, Germany), according to Pedrero et al. [25].

\subsection{Statistical Analysis}

The statistical analysis was performed with the statistical analysis system SPSS software package version 14.0 (StatSoft, Inc., Chicago, IL, USA) using GLM (General Linear Model). The five Se levels were combined with the two types of Se application in a two-factorial split-plot experimental design. The impact of the different treatments was evaluated by applying two-factorial ANOVA, while multiple comparisons of means were performed by applying the Tukey HSD test. For data expressed in percentage, the $\arcsin$ transformation before ANOVA analysis $\left(\varnothing=\arcsin (\mathrm{p} / 100)^{1 / 2}\right)$ was applied.

Principal components analysis (PCA) was conducted to examine any underlying relationship among the different Se doses and type of applications, based on the agronomic and quality parameters of curly endive at harvest. For the selection of the optimum number of principal components (PCs), factors with eigenvalues higher than 1.0 were retained. In addition, the plot of the PCs enabled the investigation of correlations between the variables of the input data set. To this end, the initial variables were projected into the subspace defined by the reduced number of PCs (first and second components) and correlated variables were identified. The PCA was performed using SPSS version 14.0 (StatSoft, Inc., Chicago, IL, USA).

\section{Results}

\subsection{Plant Se Content}

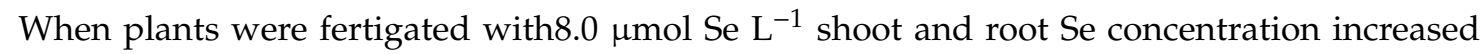
25 and 30 times, respectively, compared to non-Se fertigated plants (control). A foliar application of $8.0 \mu \mathrm{mol} \mathrm{Se} \mathrm{\textrm {L } ^ { - 1 }}$ was almost equally effective in increasing shoot and root Se concentration (approximately seven and nine times, respectively, compared to the foliar application with water). In both types of Se application, non-enriched plants showed the lowest shoot and root Se concentration (Table 1).

Table 1. Effect of Se fertigation and Se foliar spray on leaf Se concentration, root Se concentration and Se translocation factor of curly endive grown in a hydroponic system.

\begin{tabular}{|c|c|c|c|c|c|c|}
\hline \multirow{3}{*}{ 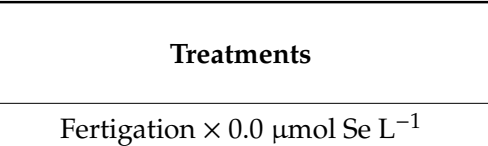 } & \multicolumn{4}{|c|}{ Se Concentration $\left(\mathrm{mg} \mathrm{kg}^{-1} \mathrm{DW}\right)$} & \multirow{2}{*}{\multicolumn{2}{|c|}{$\begin{array}{l}\text { Se Translocation Factor } \\
\text { (Shoot/Root Se ratio) }\end{array}$}} \\
\hline & \multicolumn{2}{|c|}{ Shoot } & \multicolumn{2}{|c|}{ Root } & & \\
\hline & 0.71 & $\mathrm{i}$ & 1.08 & $\mathrm{~g}$ & 0.66 & $\mathrm{~g}$ \\
\hline Fertigation $\times 1.0 \mu \mathrm{mol} \mathrm{Se} \mathrm{L^{-1 }}$ & 1.94 & $\mathrm{~g}$ & 1.25 & $\mathrm{f}$ & 1.55 & c \\
\hline Fertigation $\times 2.0 \mu \mathrm{mol} \mathrm{Se} \mathrm{L^{-1 }}$ & 3.91 & e & 1.88 & $\mathrm{e}$ & 2.08 & a \\
\hline Fertigation $\times 4.0 \mu \mathrm{mol} \mathrm{Se} \mathrm{L^{-1 }}$ & 8.83 & c & 8.87 & c & 1.00 & $\mathrm{e}$ \\
\hline Fertigation $\times 8.0 \mu \mathrm{mol} \mathrm{Se} \mathrm{L^{-1 }}$ & 17.61 & a & 33.45 & a & 0.53 & $\mathrm{~h}$ \\
\hline Foliar spray $\times 0.0 \mu \mathrm{mol} \mathrm{Se} \mathrm{L^{-1 }}$ & 0.72 & $\mathrm{i}$ & 1.07 & $\mathrm{~g}$ & 0.67 & $\mathrm{~g}$ \\
\hline Foliar spray $\times 1.0 \mu \mathrm{mol} \mathrm{Se} \mathrm{L^{-1 }}$ & 0.95 & $\mathrm{~h}$ & 1.07 & $\begin{array}{l}0 \\
\mathrm{~g}\end{array}$ & 0.89 & $\mathrm{f}$ \\
\hline Foliar spray $\times 2.0 \mu \mathrm{mol} \mathrm{Se} \mathrm{L}^{-1}$ & 2.56 & $\mathrm{f}$ & 1.26 & $\mathrm{f}$ & 2.03 & a \\
\hline Foliar spray $\times 4.0 \mu \mathrm{mol} \mathrm{Se} \mathrm{L}^{-1}$ & 5.76 & $\mathrm{~d}$ & 3.28 & $\mathrm{~d}$ & 1.75 & $\mathrm{~b}$ \\
\hline Foliar spray $\times 8.0 \mu \mathrm{mol} \mathrm{Se} \mathrm{L^{-1 }}$ & 12.67 & b & 10.42 & $\mathrm{~b}$ & 1.22 & $\mathrm{~d}$ \\
\hline \multicolumn{7}{|c|}{ Statistical significance } \\
\hline Type of application & \multicolumn{2}{|c|}{$* * *$} & \multicolumn{2}{|c|}{$* * *$} & \multicolumn{2}{|c|}{$* * *$} \\
\hline Se concentration & \multicolumn{2}{|c|}{$* * *$} & \multicolumn{2}{|c|}{$* * *$} & \multicolumn{2}{|c|}{$* * *$} \\
\hline Type of application $\times$ Se concentration & \multicolumn{2}{|c|}{$* * *$} & \multicolumn{2}{|c|}{$* * *$} & \multicolumn{2}{|c|}{$* * *$} \\
\hline
\end{tabular}

Data within a column followed by the same letter are not significantly different at $p \leq 0.05$ according to Tukey HSD Test. The significance is designated by asterisks as follows: ${ }^{* * *}$, statistically significant differences at $p$-value below 0.001 . 
Curly endive plants enriched with a dosage of $2.0 \mu \mathrm{mol} \mathrm{Se} \mathrm{L}^{-1}$ both via foliar spray and by fertigation had a Se translocation factor three times higher than the corresponding control plants (fertigated or foliar sprayed) (Table 1). The lowest Se translocation factor was observed when plants

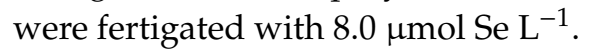

\subsection{Crop Performance}

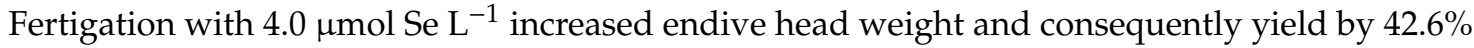
compared to fertigation without Se (control), whereas no differences were obtained when the amount of Se was either 1 or $8 \mu \mathrm{mol} \mathrm{Se} \mathrm{L}{ }^{-1}$ (Table 2). On the other hand, foliar application of 4.0 or $8.0 \mu \mathrm{mol} \mathrm{Se} \mathrm{L}{ }^{-1}$ increased yield (13.1\%and $21.8 \%$, respectively) compared to the foliar application with water. Head height, however, was affected by neither the type of application nor Se concentration (Table 2).

Table 2. Effect of Se fertigation and Se foliar spray on head fresh weight (HFW), head height (HH), stem diameter (SD), number of leaves (NL), head dry matter content (HDMC) and root dry matter content (RDMC) of curly endive grown in a hydroponic system.

\begin{tabular}{|c|c|c|c|c|c|c|c|c|c|c|c|}
\hline \multirow{2}{*}{$\frac{\text { Treatments }}{\text { Fertigation } \times 0.0 \mu \mathrm{mol} \mathrm{Se} \mathrm{L^{-1 }}}$} & \multicolumn{2}{|c|}{ HFW (g) } & \multirow{2}{*}{$\begin{array}{l}\mathbf{H H}(\mathbf{c m}) \\
27.7\end{array}$} & \multicolumn{2}{|c|}{$\mathrm{SD}(\mathrm{mm})$} & \multicolumn{2}{|c|}{ NL } & \multicolumn{2}{|c|}{ HDMC (\%) } & \multicolumn{2}{|c|}{ RDMC (\%) } \\
\hline & 219.6 & $\mathrm{e}$ & & 25.0 & c & 43.8 & $\mathrm{f}$ & 9.5 & $\mathrm{~b}$ & 2.2 & $\mathrm{~b}$ \\
\hline Fertigation $\times 1.0 \mu \mathrm{mol} \mathrm{Se} \mathrm{L}{ }^{-1}$ & 222.8 & de & 27.5 & 24.6 & c & 43.7 & $\mathrm{f}$ & 9.5 & $b$ & 2.2 & $\mathrm{~b}$ \\
\hline Fertigation $\times 2.0 \mu \mathrm{mol} \mathrm{Se} \mathrm{L^{-1 }}$ & 276.5 & $\mathrm{~b}$ & 27.4 & 27.1 & $b$ & 58.1 & c & 10.6 & a & 2.5 & a \\
\hline Fertigation $\times 4.0 \mu \mathrm{mol} \mathrm{Se} \mathrm{L^{-1 }}$ & 313.2 & a & 27.2 & 29.3 & a & 72.8 & a & 10.7 & a & 2.5 & a \\
\hline Fertigation $\times 8.0 \mu \mathrm{mol} \mathrm{Se} \mathrm{L}^{-1}$ & 214.0 & $\mathrm{e}$ & 27.5 & 23.5 & $\mathrm{~d}$ & 47.8 & e & 9.3 & $\mathrm{~b}$ & 2.2 & $\mathrm{~b}$ \\
\hline Foliar spray $\times 0.0 \mu \mathrm{mol} \mathrm{Se} \mathrm{L}^{-1}$ & 218.4 & e & 27.4 & 24.9 & $\mathrm{c}$ & 42.2 & $\mathrm{f}$ & 9.5 & $\mathrm{~b}$ & 2.2 & $\mathrm{~b}$ \\
\hline Foliar spray $\times 1.0 \mu \mathrm{mol} \mathrm{Se} \mathrm{L^{-1 }}$ & 219.2 & $\mathrm{e}$ & 27.3 & 24.1 & $\mathrm{~cd}$ & 43.0 & $\mathrm{f}$ & 9.5 & $\mathrm{~b}$ & 2.2 & $\mathrm{~b}$ \\
\hline Foliar spray $\times 2.0 \mu \mathrm{mol} \mathrm{Se} \mathrm{L^{-1 }}$ & 233.4 & $\mathrm{~d}$ & 27.9 & 27.5 & $\mathrm{ab}$ & 47.9 & $\mathrm{e}$ & 9.5 & $\mathrm{~b}$ & 2.2 & $\mathrm{~b}$ \\
\hline Foliar spray $\times 4.0 \mu \mathrm{mol} \mathrm{Se} \mathrm{L}^{-1}$ & 251.2 & c & 27.2 & 28.5 & a & 54.1 & $\mathrm{~d}$ & 9.7 & $\mathrm{~b}$ & 2.2 & $\mathrm{~b}$ \\
\hline Foliar spray $\times 8.0 \mu \mathrm{mol} \mathrm{Se} \mathrm{L}^{-1}$ & 279.2 & $\mathrm{~b}$ & 27.0 & 29.0 & $\mathrm{a}$ & 63.3 & $\mathrm{~b}$ & 10.5 & a & 2.6 & a \\
\hline \multicolumn{12}{|c|}{ Statistical significance } \\
\hline Type of application & \multicolumn{2}{|c|}{$* *$} & NS & \multicolumn{2}{|c|}{$*$} & \multicolumn{2}{|c|}{$* *$} & \multicolumn{2}{|c|}{$* *$} & \multicolumn{2}{|c|}{ NS } \\
\hline Se concentration & \multicolumn{2}{|c|}{$* * *$} & NS & \multicolumn{2}{|c|}{$* * *$} & \multicolumn{2}{|c|}{$* * *$} & \multicolumn{2}{|c|}{$* * *$} & \multicolumn{2}{|c|}{$* *$} \\
\hline Type of application $\times$ Se concentration & \multicolumn{2}{|c|}{$* * *$} & NS & \multicolumn{2}{|c|}{$* * *$} & \multicolumn{2}{|c|}{$* * *$} & \multicolumn{2}{|c|}{$* * *$} & \multicolumn{2}{|c|}{$* * *$} \\
\hline
\end{tabular}

Data within a column followed by the same letter are not significantly different at $p \leq 0.05$ according to Tukey HSD Test. ${ }^{*}$, statistically significant differences at $p \leq 0.05 ;{ }^{* *}$, statistically significant differences at $p$-value below $0.01 ;{ }^{* * *}$, statistically significant differences at p-value below 0.001 ; NS, not significant.

Se fertigated plants with $4.0 \mu \mathrm{mol} \mathrm{L} \mathrm{L}^{-1}$ and Se enriched plants via foliar spray with 4.0 and

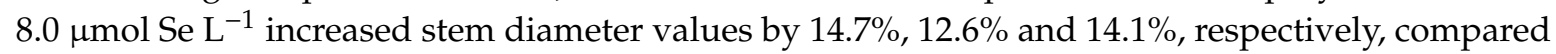
to the corresponding control plants (fertigated or foliar sprayed). The abovementioned plants were followed by those grown at a Se concentration of $2.0 \mu \mathrm{mol} \mathrm{L}{ }^{-1}$ applied via fertigation. However, stem diameter in Se enriched plants at a foliar spray dosage of $2.0 \mu \mathrm{mol} \mathrm{L}^{-1}$ did not significantly differ from the aforesaid treatments. Plants fertigated with a Se concentration of $8.0 \mu \mathrm{mol} \mathrm{L}-1$ had the lowest stem diameter value.

Fertigation of plants with $4.0 \mu \mathrm{mol} \mathrm{Se} \mathrm{L}^{-1}$ resulted in $39.8 \%$ increased number of leaves compared to fertigation without Se (control), whereas no differences were obtained when the amount of Se was $1 \mu \mathrm{mol} \mathrm{L}^{-1}$ (Table 2). Differently, non-biofortified plants and plants enriched with a dosage of $1.0 \mu \mathrm{mol} \mathrm{Se} \mathrm{L}^{-1}$, which was applied either via fertigation or foliar spray, gave the lowest values in terms of number of leaves.

With respect to head and root dry matter content, the highest percentage was recorded when plants were either fertigated with 2.0 and $4.0 \mu \mathrm{mol} \mathrm{Se} \mathrm{L}^{-1}$ or enriched with $8.0 \mu \mathrm{mol} \mathrm{L}^{-1}$ Se via foliar spray.

\subsection{Antioxidant Properties and Nutritional Value}

Fertigation with $4.0 \mu \mathrm{mol}$ Se $\mathrm{L}^{-1}$ increased SSC by $14.3 \%$ compared the control, whereas no differences were obtained when the amount of Se was either 1 or $8 \mu \mathrm{mol} \mathrm{Se} \mathrm{L}{ }^{-1}$ (Table 3). On the 
other hand, foliar application of 4.0 or $8.0 \mu \mathrm{mol} \mathrm{Se} \mathrm{L}^{-1}$ increased SSC by $6.7 \%$ and $12.5 \%$, respectively, compared to the foliar application with water.

Table 3. Effect of Se fertigation and Se foliar spray on soluble solid content (SSC), titratable acidity (TA), ascorbic acid and total phenolics of curly endive grown in a hydroponic system.

\begin{tabular}{|c|c|c|c|c|c|c|c|c|}
\hline \multirow{2}{*}{ 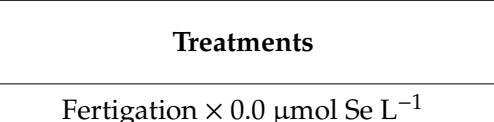 } & \multicolumn{2}{|c|}{$\operatorname{SSC}\left({ }^{\circ}\right.$ Brix $)$} & \multicolumn{2}{|c|}{ TA $(\%)$} & \multicolumn{2}{|c|}{$\begin{array}{l}\text { Ascorbic Acid } \\
\left(\mathrm{mg} \mathrm{kg}^{-1} \mathrm{fw}\right)\end{array}$} & \multicolumn{2}{|c|}{$\begin{array}{l}\text { Total Phenolic (mg of } \\
\text { Caffeic Acid } \mathrm{g}^{-1} \text { f.w.) }\end{array}$} \\
\hline & 4.2 & c & 0.6 & e & 76.1 & $\mathrm{e}$ & 0.56 & $\mathrm{~g}$ \\
\hline Fertigation $\times 1.0 \mu \mathrm{mol} \mathrm{Se} \mathrm{L}^{-1}$ & 4.4 & $\mathrm{bc}$ & 0.7 & $\mathrm{~d}$ & 82.6 & $\mathrm{~d}$ & 0.62 & $\mathrm{fg}$ \\
\hline Fertigation $\times 2.0 \mu \mathrm{mol} \mathrm{Se} \mathrm{L}^{-1}$ & 4.6 & $\mathrm{~b}$ & 0.8 & c & 94.9 & c & 0.77 & $\mathrm{~d}$ \\
\hline Fertigation $\times 4.0 \mu \mathrm{mol} \mathrm{Se} \mathrm{L^{-1 }}$ & 4.9 & a & 1.0 & $\mathrm{~b}$ & 101.4 & $\mathrm{~b}$ & 0.89 & $\mathrm{~b}$ \\
\hline Fertigation $\times 8.0 \mu \mathrm{mol} \mathrm{Se} \mathrm{L^{-1 }}$ & 4.3 & c & 1.1 & a & 104.7 & a & 0.95 & a \\
\hline Foliar spray $\times 0.0 \mu \mathrm{mol} \mathrm{Se} \mathrm{L}^{-1}$ & 4.2 & c & 0.6 & e & 75.1 & $\mathrm{e}$ & 0.55 & $\mathrm{~g}$ \\
\hline Foliar spray $\times 1.0 \mu \mathrm{mol} \mathrm{Se} \mathrm{L^{-1 }}$ & 4.3 & c & 0.6 & $\mathrm{e}$ & 75.3 & e & 0.57 & $\mathrm{~g}$ \\
\hline Foliar spray $\times 2.0 \mu \mathrm{mol} \mathrm{Se} \mathrm{L}^{-1}$ & 4.3 & c & 0.6 & $\mathrm{e}$ & 82.0 & $\mathrm{~d}$ & 0.64 & f \\
\hline Foliar spray $\times 4.0 \mu \mathrm{mol} \mathrm{Se} \mathrm{L^{-1 }}$ & 4.5 & $\mathrm{~b}$ & 0.7 & $\mathrm{~d}$ & 94.2 & c & 0.73 & $\mathrm{e}$ \\
\hline Foliar spray $\times 8.0 \mu \mathrm{mol} \mathrm{Se} \mathrm{L^{-1 }}$ & 4.8 & $\mathrm{a}$ & 1.0 & $\mathrm{~b}$ & 100.4 & $\mathrm{~b}$ & 0.85 & c \\
\hline \multicolumn{9}{|c|}{ Statistical significance } \\
\hline Type of application & \multicolumn{2}{|c|}{ NS } & \multicolumn{2}{|c|}{$* * *$} & \multicolumn{2}{|c|}{$* * *$} & \multicolumn{2}{|c|}{$* * *$} \\
\hline Se concentration & \multicolumn{2}{|c|}{$* * *$} & \multicolumn{2}{|c|}{$* * *$} & \multicolumn{2}{|c|}{$* * *$} & \multicolumn{2}{|c|}{$* * *$} \\
\hline Type of application $\times$ Se concentration & \multicolumn{2}{|c|}{$* * *$} & \multicolumn{2}{|c|}{$* * *$} & \multicolumn{2}{|c|}{$* * *$} & \multicolumn{2}{|c|}{$* * *$} \\
\hline
\end{tabular}

Data within a column followed by the same letter are not significantly different at $p \leq 0.05$ according to Tukey HSD

Test. ***, statistically significant differences at $p \leq 0.001$; NS, not significant.

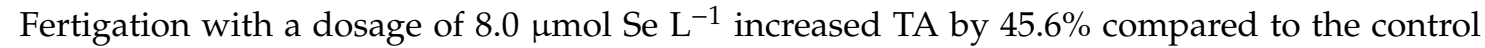
(Table 3). Similarly, foliar application of $8.0 \mu \mathrm{mol} \mathrm{Se} \mathrm{L}^{-1}$ increased TA by $40.0 \%$ compared to the foliar application with water. The lowest TA value was recorded in non-biofortified plants (regardless of the

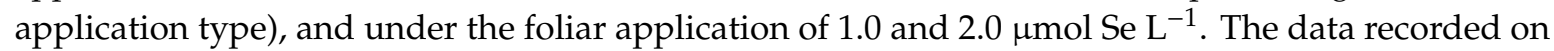
ascorbic acid support the trend established for TA (Table 3).

Curly endive plants fertigated with a Se concentration of $8.0 \mu \mathrm{mol} \mathrm{L} \mathrm{L}^{-1}$ had a total phenolic content $41.1 \%$ higher than those fertigated without Se (control), whereas no differences were detected when the amount of Se was either 1.0 or $0.0 \mu \mathrm{mol} \mathrm{Se} \mathrm{L}{ }^{-1}$ (Table 3). Equally, foliar application of $8.0 \mu \mathrm{mol} \mathrm{Se} \mathrm{L}{ }^{-1}$ increased total phenolic by $35.3 \%$ compared to the foliar application with water.

Leaves from curly endive plants grown without Se enrichment (fertigation $\times 0.0 \mu \mathrm{mol} \mathrm{Se} \mathrm{L^{-1 }}$ and

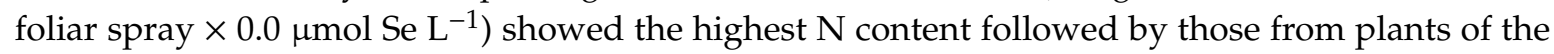

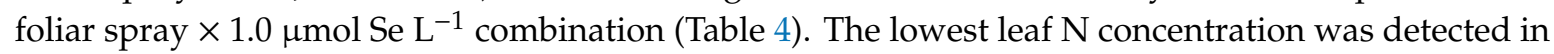
plants fertigated with $8.0 \mu \mathrm{mol} \mathrm{Se} \mathrm{L}^{-1}$.

Type of application and Se concentration did not significantly affect $\mathrm{P}$ and $\mathrm{K}$ leaf concentrations (Table 4). Regardless of the type of application, the non-biofortified plants and plants enriched with $1.0 \mu \mathrm{molSe} \mathrm{L}^{-1}$ had the highest leaf Ca concentrations, followed by those from the combination of foliar spray $\times 2.0 \mu \mathrm{mol} \mathrm{Se} \mathrm{L}{ }^{-1}$. Plants fertigated with a Se concentration of $8.0 \mu \mathrm{mol} \mathrm{L}^{-1}$ showed the lowest leaf Ca content. Neither type of application nor Se concentration affected $\mathrm{Mg}$ leaf concentration

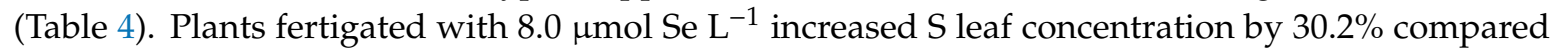
to those fertigated without Se (control). Likewise, foliar application of $8.0 \mu \mathrm{mol} \mathrm{Se} \mathrm{L^{-1 }}$ resulted in increased S leaf concentration (16.8\%) compared to the foliar application with water. No differences

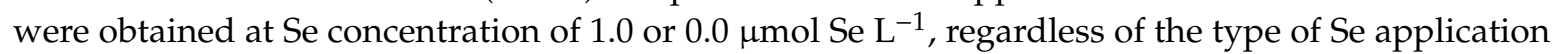
(Table 4). Non-enriched plants showed the highest value in terms of S/Se ratio, followed by those grown at a Se concentration of $1.0 \mu \mathrm{mol} \mathrm{L}{ }^{-1}$ applied via foliar spray, which in turn showed a higher value than plants fertigated with $1.0 \mu \mathrm{mol} \mathrm{Se} \mathrm{L}^{-1}$ (Table 4). The lowest $\mathrm{S} / \mathrm{Se}$ ratio was recorded from

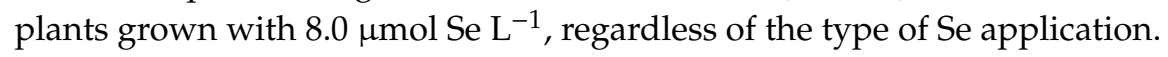


Table 4. Effect of Se fertigation and Se foliar spray on N, P, K, Ca, Mg, S and S/Se ratio of curly endive grown in a hydroponic system.

\begin{tabular}{|c|c|c|c|c|c|c|c|c|c|c|c|}
\hline \multirow{2}{*}{ 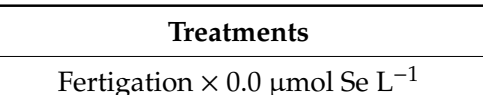 } & \multicolumn{2}{|c|}{$\mathbf{N}\left(\mathrm{mg} \mathrm{g}^{-1} \mathrm{DW}\right)$} & \multirow{2}{*}{$\frac{\mathbf{P}\left(\mathrm{mg} \mathrm{g}^{-1} \mathrm{DW}\right)}{0.66}$} & \multirow{2}{*}{$\begin{array}{l}\mathrm{K}\left(\mathrm{mg} \mathrm{g}^{-1} \mathrm{DW}\right) \\
3.11\end{array}$} & \multicolumn{2}{|c|}{$\mathrm{Ca}\left(\mathrm{mg} \mathrm{g}^{-1} \mathrm{DW}\right)$} & \multirow{2}{*}{$\begin{array}{l}\mathbf{M g}\left(\mathrm{mg} \mathrm{g}^{-1} \mathbf{D W}\right) \\
0.33\end{array}$} & \multicolumn{2}{|c|}{$S\left(\mathrm{mg} \mathrm{g}^{-1} \mathrm{DW}\right)$} & \multicolumn{2}{|c|}{ S/Se Ratio } \\
\hline & 5.60 & $\mathrm{a}$ & & & 0.71 & $\mathrm{a}$ & & 3.82 & e & 5.35 & $\mathrm{a}$ \\
\hline Fertigation $\times 1.0 \mu \mathrm{mol} \mathrm{Se} \mathrm{L^{-1 }}$ & 5.24 & $\mathrm{bc}$ & 0.65 & 3.07 & 0.70 & $\mathrm{a}$ & 0.33 & 3.86 & $\mathrm{e}$ & 1.99 & c \\
\hline Fertigation $\times 2.0 \mu \mathrm{mol} \mathrm{Se} \mathrm{L^{-1 }}$ & 4.99 & c & 0.63 & 3.11 & 0.55 & $\mathrm{~cd}$ & 0.33 & 4.24 & c & 1.09 & e \\
\hline Fertigation $\times 4.0 \mu \mathrm{mol} \mathrm{Se} \mathrm{L}^{-1}$ & 4.32 & $\mathrm{~d}$ & 0.65 & 3.04 & 0.52 & $\mathrm{~d}$ & 0.35 & 4.67 & $\mathrm{~b}$ & 0.53 & $\mathrm{~g}$ \\
\hline Fertigation $\times 8.0 \mu \mathrm{mol} \mathrm{Se} \mathrm{L}^{-1}$ & 3.39 & $\mathrm{f}$ & 0.61 & 3.07 & 0.42 & $\mathrm{f}$ & 0.34 & 5.47 & a & 0.31 & $\mathrm{~h}$ \\
\hline Foliar spray $\times 0.0 \mu \mathrm{mol} \mathrm{Se} \mathrm{L^{-1 }}$ & 5.60 & $\mathrm{a}$ & 0.64 & 3.10 & 0.72 & $\mathrm{a}$ & 0.36 & 3.80 & $\mathrm{e}$ & 5.30 & $\mathrm{a}$ \\
\hline Foliar spray $\times 1.0 \mu \mathrm{mol} \mathrm{Se} \mathrm{L}^{-1}$ & 5.40 & $\mathrm{~b}$ & 0.68 & 3.12 & 0.73 & a & 0.32 & 3.79 & $\mathrm{e}$ & 4.00 & $\mathrm{~b}$ \\
\hline Foliar spray $\times 2.0 \mu \mathrm{mol} \mathrm{Se} \mathrm{L}-1$ & 5.24 & $\mathrm{bc}$ & 0.64 & 3.05 & 0.66 & $\mathrm{~b}$ & 0.35 & 3.74 & e & 1.46 & d \\
\hline Foliar spray $\times 4.0 \mu \mathrm{mol} \mathrm{Se} \mathrm{L}^{-1}$ & 4.91 & c & 0.66 & 3.06 & 0.58 & c & 0.32 & 4.07 & $\mathrm{~d}$ & 0.71 & $\mathrm{f}$ \\
\hline Foliar spray $\times 8.0 \mu \mathrm{mol} \mathrm{Se} \mathrm{L}^{-1}$ & 4.00 & e & 0.65 & 3.08 & 0.49 & e & 0.36 & 4.57 & $\mathrm{~b}$ & 0.36 & $\mathrm{~h}$ \\
\hline \multicolumn{12}{|c|}{ Statistical significance } \\
\hline Type of application & \multicolumn{2}{|c|}{$* * *$} & NS & NS & \multicolumn{2}{|c|}{$* * *$} & NS & \multicolumn{2}{|c|}{$* * *$} & \multicolumn{2}{|c|}{$* * *$} \\
\hline Se concentration & \multicolumn{2}{|c|}{$* * *$} & NS & NS & \multicolumn{2}{|c|}{$* * *$} & NS & \multicolumn{2}{|c|}{$* * *$} & \multicolumn{2}{|c|}{$* * *$} \\
\hline Type of application $\times$ Se concentration & \multicolumn{2}{|c|}{$* * *$} & NS & NS & \multicolumn{2}{|c|}{ * } & NS & \multicolumn{2}{|c|}{$* * *$} & \multicolumn{2}{|c|}{$* * *$} \\
\hline
\end{tabular}

Data within a column followed by the same letter are not significantly different at $p \leq 0.05$ according to Tukey HSD Test. * , statistically significant differences at $p \leq 0.05 ; * * *$, statistically significant differences at $p$-value below 0.001; NS, not significant. 


\subsection{Principal Component Analysis}

The outcomes of the principal component analysis showed four main components (PCs) with eigenvalues higher than 1.00 (Table 5), accounting for $56.00 \%, 21.54 \%, 8.49 \%$ and $6.39 \%$ of the total variance, respectively.

Table 5. Correlation of variables to the factors of the principal components analysis (PCA) based on factor loadings.

\begin{tabular}{ccccc}
\hline Variable & PC1 & PC2 & PC3 & PC4 \\
\hline Head weight & $\mathbf{0 . 7 3 1}$ & $\mathbf{0 . 6 5 4}$ & 0.064 & 0.053 \\
Head height & -0.491 & -0.338 & $-\mathbf{0 . 6 0 9}$ & 0.226 \\
No. of leaves & $\mathbf{0 . 8 4 6}$ & 0.494 & 0.067 & 0.027 \\
Stem diameter & 0.571 & $\mathbf{0 . 7 2 1}$ & -0.217 & -0.003 \\
Head dry matter & 0.665 & $\mathbf{0 . 6 5 8}$ & 0.173 & 0.159 \\
Root dry matter & $\mathbf{0 . 7 0 1}$ & 0.520 & 0.255 & 0.325 \\
SSC & $\mathbf{0 . 8 4 4}$ & 0.511 & 0.077 & 0.004 \\
TA & $\mathbf{0 . 9 4 0}$ & -0.279 & 0.120 & 0.037 \\
Ascorbic acid & $\mathbf{0 . 9 7 2}$ & -0.115 & -0.036 & -0.168 \\
Total phenolic & $\mathbf{0 . 9 7 2}$ & -0.201 & 0.001 & -0.093 \\
N & $\mathbf{- 0 . 8 9 4}$ & 0.416 & -0.047 & 0.068 \\
P & -0.507 & 0.536 & 0.334 & -0.387 \\
K & -0.574 & -0.012 & $\mathbf{0 . 6 0 6}$ & 0.076 \\
Ca & $\mathbf{- 0 . 9 4 7}$ & 0.249 & 0.028 & 0.088 \\
Mg & 0.338 & -0.005 & -0.242 & $\mathbf{0 . 8 4 0}$ \\
S & $\mathbf{0 . 8 3 4}$ & -0.522 & 0.148 & -0.026 \\
Se shoot & $\mathbf{0 . 8 6 0}$ & -0.468 & 0.063 & -0.047 \\
Se root & 0.632 & $-\mathbf{0 . 7 6 7}$ & 0.073 & -0.026 \\
Se translocation factor & 0.061 & 0.549 & $-\mathbf{0 . 6 6 9}$ & -0.288 \\
Eigenvalue & $\mathbf{- 0 . 8 5 4}$ & -0.028 & 0.369 & 0.309 \\
\hline Variance \% & 11.201 & 4.308 & 1.698 & 1.279 \\
Cumulative \% & 56.006 & 21.539 & 8.488 & 6.393 \\
\hline Valus & 56.006 & 77.545 & 86.033 & 92.426 \\
\hline in bold with & & & & \\
\hline
\end{tabular}

Values in bold within the same factor indicate the variable with the largest correlation.

This indicated that the initial twenty variables could be expressed as a linear combination of four PCs, explaining $92.43 \%$ of the total variance. PC1 was positively related to head weight, number of leaves, root dry matter, SSC, TA, ascorbic acid, total phenolic, S, and Se shoot, and negatively correlated to $\mathrm{N}, \mathrm{Ca}$, and S/Se ratio; PC2 was mainly positively related to head weight, stem diameter, and head dry matter, and negatively related to Se root; $\mathrm{PC} 3$ was mainly positively correlated to K, and negatively related to head height and Se translocation factor; and PC4 was mainly positively related to $\mathrm{Mg}$ content (Table 5).

The projection of the original variables on the plane of the two first PCs could clearly illustrate such a relationship, as shown in the plot of loadings (Figure 1).

The discrimination of the various doses of Se supplied via different type of application (fertigation and foliar spray) of curly endive can be visualized in the plot of scores (Figure 2), where five clusters could be distinguished.

The controls (fertigation_0.0 and foliar spray_0.0), fertigation_1.0, foliar spray_1.0 and foliar spray_2.0 are positioned on the left side of the plot of loadings and other samples are positioned on its right side, with fertigation_8.0 situated in the bottom-right position (Figure 2). 


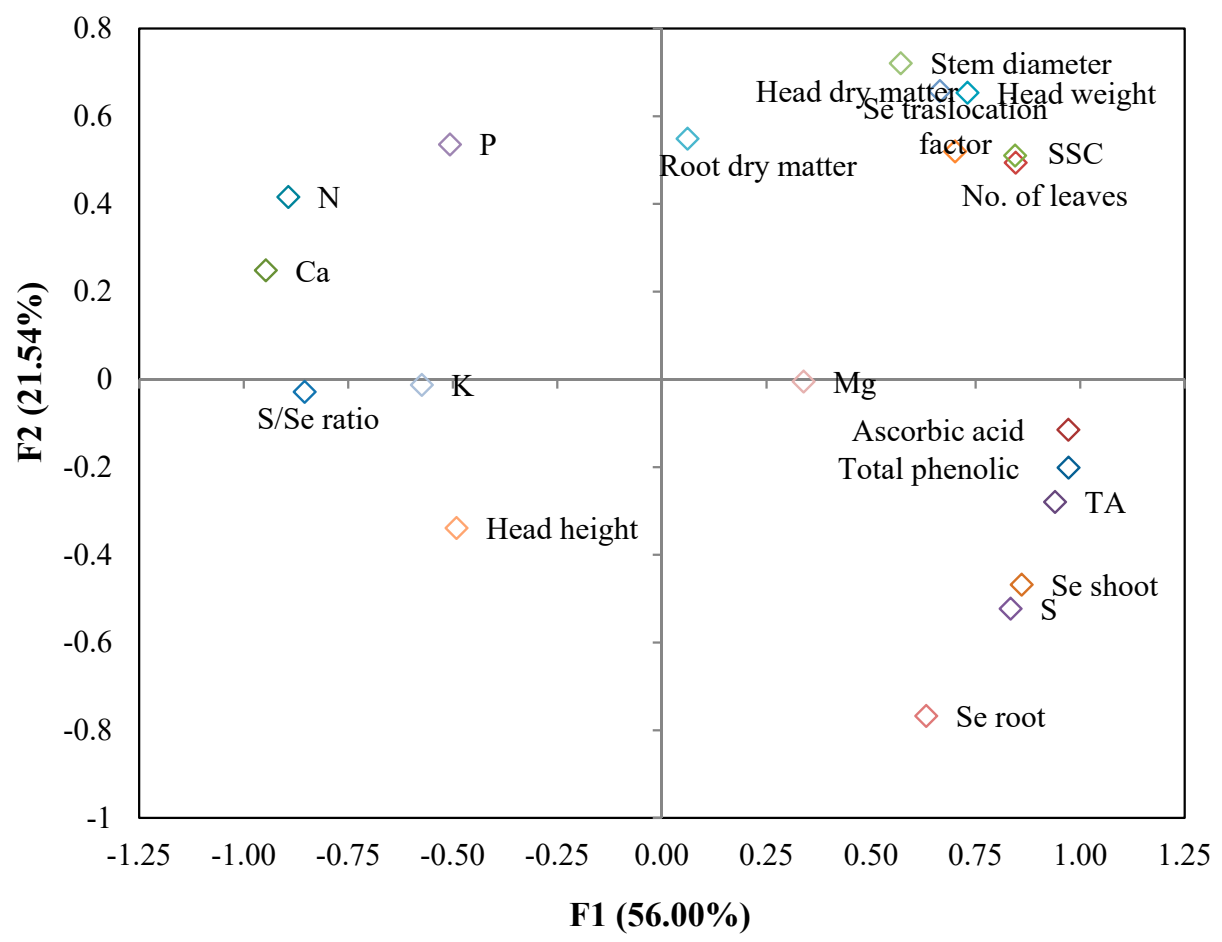

Figure 1. Plot of loadings: agronomic and qualitative parameters of curly endive at harvest.

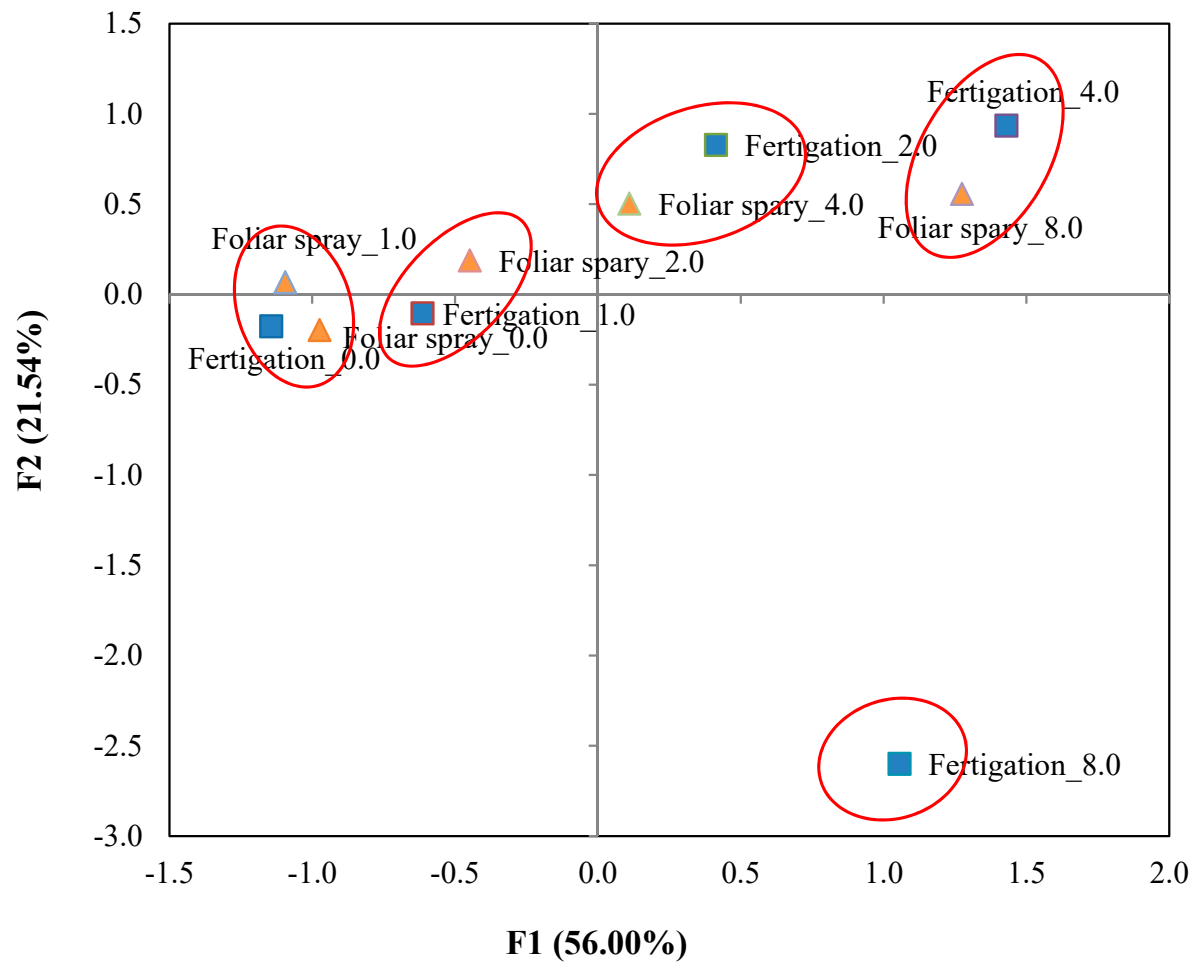

Figure 2. Plot of scores (trial) formed by the first two principal components from the PCA analysis. Fertigation_0.0,Fertigation_1.0, Fertigation_2.0, Fertigation_4.0 and Fertigation_8.0: curly endive plants grown in a hydroponic floating system using nutrient solutions with five levels of selenium $(0,1.0$, 2.0, 4.0 and $8.0 \mu \mathrm{mol} \mathrm{L}{ }^{-1}$, respectively) distributed via fertigation; Foliar spray_0.0, Foliar spray_1.0, Foliar spray_2.0, Foliar spray_4.0 and Foliar spray_8.0: curly endive plants grown in a hydroponic floating system using nutrient solutions with five levels of selenium $\left(0,1.0,2.0,4.0\right.$ and $8.0 \mu \mathrm{mol} \mathrm{L}^{-1}$, respectively) distributed via foliar spray. 


\section{Discussion}

The connection between food intake and health is a current and continuously expanding research topic as substantial outcomes indicate that food compounds can affect physiological processes in humans. Thus, nutraceutical and functional food is of great interest in prevention and cure of human illnesses. For humans, Se is an essential trace element, and some organic forms such as methylselenocysteine (MeSeCys) seem to be notably effective source of dietary Se. Hatfield et al. [26], Roman et al. [27] and Malagoli et al. [28] reported that low intake of Se in the diet may cause a number of diseases, including heart diseases, hypothyroidism, reduced male fertility, weakened immune system and enhanced susceptibility to infections and cancer. This study demonstrated that Se application rate and type (fertigation or foliar spray) can significantly affect yield, functional properties (phenolics and ascorbic acid) and mineral composition of curly endive grown in a hydroponic system.

We found a significant decrease in head weight, head height, stem diameter, number of leaves, head dry matter and root dry matter at low $\left(1.0 \mu \mathrm{mol} \mathrm{Se} \mathrm{L}^{-1}\right)$ Se concentration or over $4.0 \mu \mathrm{mol} \mathrm{Se} \mathrm{\textrm {L } ^ { - 1 }}$ when Se was distributed by fertigation. In previous research [29], the Se supply to spinach plants resulted in a leaf yield increase. However, an increase in plant growth by Se at low doses has been previously documented in different plant species, including Se hyper-accumulators (some species of the genera Astragalus, Xylorrhiza and Stanleyea and members of the Brassicaceae such as black mustard (Brassica nigra L.) and broccoli (Brassica oleracea botrytis L.)) and non-hyper-accumulators (ryegrass, lettuce, potato, duckweed, and tomato) $[8,16,30,31]$. On the other hand, the Se enriched plants by foliar spray increased plant growth under the highest dosage $\left(8.0 \mu \mathrm{mol} \mathrm{Se} \mathrm{L}^{-1}\right)$. This result may be related to the longer plant-Se contact time promoted by the nutrient solution application. Therefore, this effect would also indicate that Se distributed in a soilless system via fertigation accumulates continuously and cumulatively into the plant tissues. However, application of Se via the nutrient solution at the rate of $8 \mathrm{mM}$ led to a reduction in plant growth. This can be ascribed to a toxic effect of Se. As reported by Hajiboland and Amjad [32], the toxic effect of Se on plants may result mainly from interferences of this element with S metabolism and from replacing the S-amino acids with corresponding Se-amino acids and their subsequent incorporation into proteins.

Leaf Se concentration in enriched plants ranged from $0.95 \mathrm{mg} \mathrm{kg}^{-1} \mathrm{DW}$ to $12.67 \mathrm{mg} \mathrm{kg}^{-1} \mathrm{DW}$. Ramos et al. [17] reported that Se concentration in enriched lettuce plants, irrigated with seven Se concentrations $(0,2,4,8,16,32$, and $64 \mu \mathrm{mol} \mathrm{L}-1)$, ranged from 0.0 to about $23.0 \mathrm{mg} \mathrm{kg}^{-1} \mathrm{DW}$. Ferrarese et al. [33] who conducted a study on Se biofortification of spinach plants in a floating system with a Se concentration of $0.0,2.6,3.9$, and $5.2 \mu \mathrm{M}$ of Se, reported a variation in leaf Se concentration from approximately 1.0 to $15.5 \mu \mathrm{g} \mathrm{g}^{-1} \mathrm{DW}$. Ávila et al. [34], who studied the impact of selenium supply on Se-methylselenocysteine and glucosinolate accumulation in selenium-biofortified Brassica sprouts, found that Brassica species (Broccoli, Cauliflower, green cabbage, Chinese cabbage, kale and brussels sprouts) treated with $50 \mu \mathrm{M}$ of $\mathrm{Na}_{2} \mathrm{SO}_{4}$ had a total Se content that ranged from approximately 60.0 to over $300 \mu \mathrm{g} \mathrm{g}^{-1} \mathrm{DW}$ in brussels sprout and kale, respectively. The recommended daily amount (RDA) for adult men and women is 55-70 $\mu \mathrm{g}$ [35]. Considering that curly endive contains about $90 \%$ of water, the Se concentration in the edible part would be sufficient to cover the human physiological needs of this element by consuming $47.4 \mathrm{~g}$ day $^{-1}$ of curly endive grown under hydroponic conditions and treated with $8 \mu \mathrm{mol} \mathrm{L} \mathrm{L}^{-1}$ of selenate which was applied via foliar spray. Thus, our results support the viability of the use of curly endive crops in biofortification programs.

Due to their chemical similarities, Se and S compete for the same transporters, consequently, their metabolisms are closely interrelated [8]. Therefore, in our study, S accumulation was also determined. Our results show an increase in S concentration under the application of $1.0 \mu \mathrm{mol} \mathrm{Se} \mathrm{\textrm {L } ^ { - 1 }}$

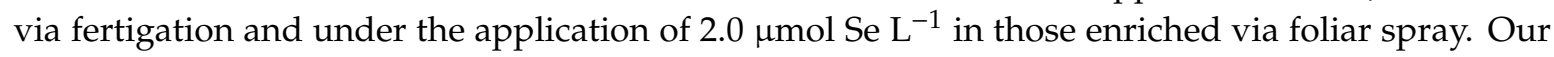
findings are in accordance with those of Hawrylak-Nowak [1], Feist and Parker [36], Suarez et al. [37], White et al. [38] and Ríos et al. [39] who found that increasing selenate concentrations in the root area increases $\mathrm{S}$ concentration in shoots in Lactuca sativa, Stanleya pinnata, some Brassica species and Arabidopsis thaliana. Our results are also in accordance with those of Boldrin et al. [40], who, by 
investigating rice biofortification by soil and foliar application of selenium, found that both types of application determined an increase in $\mathrm{S}$ concentration. The interactive effects between Se and $\mathrm{S}$ nutrition were also detected in Arabidopsis thaliana by White et al. [38]. The results of this study indicate that selenate supply can promote sulfate accumulation in the shoots, possibly by preventing a reduction in the abundance and/or activity of sulfate transporters by sulfate and its metabolites.

Nowadays, there is increasing evidence demonstrating the additive and synergetic effects of antioxidative compounds from vegetables on human health, as they can reduce the risk of many pathologies related to oxidative stress [41]. Although several studies have been conducted on the application of Se in vegetable plants, few have confirmed the positive effect of this trace element on the antioxidant capacity. Our results show that regardless of the type of application (fertigation or foliar spray) total phenolic compounds increased linearly as Se concentration applied increased. Our findings are consistent with those obtained by Ríos et al. [39] who, by investigating the biofortification of Se and induction of the antioxidant capacity in lettuce plants, found that Se-treatment augments antioxidant compounds in the leaves. Our outcomes are also in accordance with those reported by Schiavon et al. [16], who, by studying the effect of selenium fertilization on the alteration of chemical composition and antioxidant constituents of tomato, found that the supplementation of Se at low doses to tomato plants cultivated hydroponically exerted beneficial effects on the plants with respect to biosynthesis of antioxidant compounds implied in plant development and responses to stresses. Similarly, in our study, production of antioxidant compounds (total polyphenols and ascorbic acid)

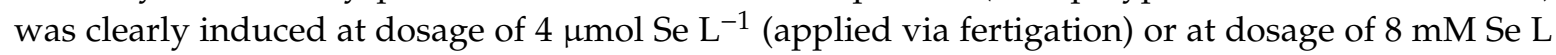
(applied via foliar spray) implying a possible beneficial effect of this trace element in curly endive plants. At a dosage of $8 \mathrm{mM} \mathrm{Se} \mathrm{L}^{-1}$ (applied via fertigation), antioxidant compounds still increased, although in this case and perhaps due to the toxic effect of Se, the plants showed a reduction in head dry matter content. Dixon and Paiva [42] and Moglia et al. [43] reported that the mechanism of action leading to an accumulation in phenolic compounds has been generally associated to a plant stress condition, and our results might confirm this theory. Lee et al. [44] and $\mathrm{Xu}$ et al. [45] revealed that Se induces the accumulation of ascorbic acid. Our results indicate that irrespective of the type of application, the incremental rise in the Se dosage to the curly endive plants led to the synthesis of ascorbic acid. Our findings are in accordance with those of Ríos et al. [39], who found that ascorbic acid content in lettuce leaves increased gradually with increasing Se supplied to the plants, via fertigation or via foliar spray.

Increasing Se concentration both via fertigation and by foliar enhanced SSC, consistently with the reports of Golubkina et al. [46] who recorded the increase of leaf soluble solids in Brassica juncea upon Se foliar application. A significant interaction was found between the type of application and Se application rate in terms of TA. Increasing Se concentration resulted in higher TA, with foliar spray application resulting superior than fertigation.

Studies have indicated that a consumption of vegetables with high $\mathrm{NO}_{3}{ }^{-}$content poses threat to human health because ingested $\mathrm{NO}_{3}{ }^{-}$could be converted to nitrite, a toxic carcinogen, causing cancers and methemoglobinemia $[47,48]$. Our results on $\mathrm{N}$ content are in line with the outcomes of Lei et al. [49], who, by investigating on the effects of exogenous Se on $\mathrm{NO}_{3}{ }^{-}$uptake and transport, assimilation enzyme activities and photosynthetic capacity of lettuce grown hydroponically, found that the supplementation of Se decreased $\mathrm{NO}_{3}{ }^{-}$accumulation in the leaves by increasing the efflux of $\mathrm{NO}_{3}{ }^{-}$from the root, inhibiting the translocation of $\mathrm{NO}_{3}{ }^{-}$from the root to the shoot, and inducing the assimilation of $\mathrm{NO}_{3}{ }^{-}$. The type of application and Se concentration had no influence on $\mathrm{Mg}, \mathrm{P}$ and K leaf concentration whereas, increasing Se concentration either via fertigation or by spraying resulted in lower Ca leaf content.

\section{Conclusions}

Se biofortification of curly endive significantly influenced plant yield, leaf overall composition and leaf nutraceutical compounds. Compared with the non-biofortified plants, a Se implementation 
of $4 \mu \mathrm{mol} \mathrm{Se} \mathrm{L}^{-1}$ via fertigation or of $8 \mu \mathrm{mol} \mathrm{Se} \mathrm{L}^{-1}$ by foliar spraying successfully enhanced plant production performance, accumulation of SSC as well as some antioxidant compounds such as ascorbic acid and total phenolic. Our findings also reveal that the enrichment of Se in the nutrient solution was not detrimental up to 4 and $8 \mu \mathrm{mol} \mathrm{Se} \mathrm{L}^{-1}$ for fertigation and foliar spray application, respectively. Furthermore, our results indicate that a consumption of $47.4 \mathrm{~g} \mathrm{day}^{-1}$ of Se-enriched curly endive leaves from plants subjected to an $8 \mu \mathrm{mol} \mathrm{L} \mathrm{L}^{-1}$ selenate application via foliar spray would be sufficient to cover the human physiological needs. Thus, taken together, we can conclude that Se biofortification of hydroponic grown curly endive plants represents an attractive and easy opportunity for increasing the Se concentration in human diets.

Author Contributions: L.S., F.D. and G.I. conceived and designed the research; L.S. and G.N analyzed the data; L.S. wrote the manuscript; G.I. and G.N. helped draft the manuscript; and C.D.P. performed laboratory analytical determination.

Funding: This research received no external funding.

Conflicts of Interest: The authors declare no conflict of interest.

\section{References}

1. Hawrylak-Nowak, B. Comparative effects of selenite and selenate on growth and selenium accumulation in lettuce plants under hydroponic conditions. Plant Growth Regul. 2013, 70, 149-157. Available online: https://link.springer.com/article/10.1007/s10725-013-9788-5 (accessed on 14 February 2019). [CrossRef]

2. White, P.J.; Broadley, M.R. Biofortification of crops with seven mineral elements often lacking human diets-iron, zinc, copper, calcium, magnesium, selenium and iodine. New Phytol. 2009, 182, 49-84. Available online: https://www.ncbi.nlm.nih.gov/pubmed/19192191 (accessed on 14 February 2019). [CrossRef]

3. Moncada, A.; Miceli, A.; Sabatino, L.; Iapichino, G.; D'Anna, F.; Vetrano, F. Effect of molybdenum rate on yield and quality of lettuce, escarole, and curly endive grown in a floating system. Agronomy 2018, 8, 171. Available online: https://www.mdpi.com/2073-4395/8/9/171/htm (accessed on 14 February 2019). [CrossRef]

4. Sabatino, L.; D'Anna, F.; D'Anna, F.; Iapichino, G.; Moncada, A.; D'Anna, E.; De Pasquale, C. Interactive effects of genotype and molybdenum supply on yield and overall fruit quality of tomato. Front. Plant Sci. 2019, 9, 1922. Available online: https://www.frontiersin.org/articles/10.3389/fpls.2018.01922/full (accessed on 14 February 2019). [CrossRef] [PubMed]

5. U.S. Department of Agriculture. Dietary Reference Intakes: Elements. 2003. Available online: http: //warp.nal.usda.gov/fnic/etextA/000105.html (accessed on 14 February 2019).

6. Kieliszek, M.; Blažejak, S. Selenium: Significance, and outlook for supplementation. Nutrition 2013, 29, 713-718. Available online: https://www.ncbi.nlm.nih.gov/pubmed/23422539 (accessed on 14 February 2019). [CrossRef]

7. Gissel-Nielsen, G.; Gupta, U.C.; Lamand, M.; Westermarck, T. Selenium in soils and plants and its importance in livestock and human nutrition. Adv. Agron. 1984, 37, 397-460. Available online: https://www.sciencedirect. com/science/article/pii/S0065211308604599 (accessed on 14 February 2019).

8. Marschner, P. Marschner's Mineral Nutrition of Higher Plants, 3rd ed.; Elsevier/Academic Press: Amsterdam, The Netherlands, 2012.

9. Rayman, M.P. Selenium and human health. Lancet 2012, 379, 1256-1268. Available online: https://www.ncbi. nlm.nih.gov/pubmed/22381456 (accessed on 14 February 2019). [CrossRef]

10. Drutel, A.; Archambeaud, F.; Caron, P. Selenium and the thyroid gland: More good news for clinicians. Clin. Endocrinol. (Oxford) 2013, 78, 155-164. Available online: https://www.ncbi.nlm.nih.gov/pubmed/ 23046013 (accessed on 14 February 2019). [CrossRef] [PubMed]

11. Mistry, H.D.; Broughton Pipkin, F.; Redman, C.W.; Poston, L. Selenium in reproductive health. Am. J. Obstet. Gynecol. 2012, 206, 21-30. Available online: https://www.ncbi.nlm.nih.gov/pubmed/21963101 (accessed on 14 February 2019). [CrossRef]

12. Dennert, G.; Zwahlen, M.; Brinkman, M.; Vinceti, M.; Zeegers, M.P.; Horneber, M. Selenium for preventing cancer. Cochrane Database Syst. Rev. 2011, 5, CD005195. [CrossRef] 
13. Chen, L.; Yang, F.; Xu, J.; Yun, H.; Hu, Q.; Zhang, Y.; Pan, G. Determination of selenium concentration of rice in China and effect of fertilization of selenite and selenate on Se content of rice. J. Agric. Food Chem. 2002, 50, 5128-5130. Available online: https://www.ncbi.nlm.nih.gov/pubmed/12188618 (accessed on 14 February 2019). [CrossRef]

14. Hawkesford, M.J.; Zhao, F.J. Strategies for increasing the selenium content of wheat. J. Cereal Sci. 2007, 46, 282-292. Available online: https://www.sciencedirect.com/science/article/pii/S0733521007000379 (accessed on 14 February 2019). [CrossRef]

15. Lyons, G.H.; Stangoulis, J.C.R.; Graham, R.D. Exploiting micronutrient interaction to optimize biofortification programs: The case for inclusion of selenium and iodine in the Harvest Plust programs. Nutr. Rev. 2004, 62, 247-252. Available online: https://www.ncbi.nlm.nih.gov/pubmed/15291398 (accessed on 14 February 2019). [CrossRef]

16. Schiavon, M.; dall'Acqua, S.; Mietto, A.; Pilon-Smits, E.A.H.; Sambo, P.; Masi, A.; Malagoli, M. Selenium Fertilization Alters the Chemical Composition and Antioxidant Constituents of Tomato (Solanum lycopersicon L.). J. Agric. Food Chem. 2013, 61, 10542-10554. Available online: https://www.ncbi.nlm.nih.gov/pubmed/24079300 (accessed on 14 February 2019). [CrossRef]

17. Ramos, S.J.; Faquin, V.; Guilherme, L.R.G.; Castro, E.M.; Ávila, F.W.; Carvalho, G.S.; Bastos, C.E.A.; Oliveira, C. Selenium biofortification and antioxidant activity in lettuce plants fed with selenate and selenite. Plant Soil Environ. 2010, 56, 584-588. Available online: https://www.agriculturejournals.cz/publicFiles/31992.pdf (accessed on 14 February 2019). [CrossRef]

18. Koudela, M.; Petř́ková, K. Nutritional composition and yield of endive cultivars-Cichorium endivia L. Hort. Sci. 2007, 34, 6-10. Available online: https://www.agriculturejournals.cz/publicFiles/00115.pdf (accessed on 14 February 2019). [CrossRef]

19. Sonneveld, C.; Voogt, W. Plant Nutrition of Greenhouse Crops; Springer: New York, NY, USA, 2009.

20. Goto, E.; Both, A.J.; Albright, L.D.; Langhans, R.W.; Leed, A.R. Effect of dissolved oxygen concentration on lettuce rowth in floating hydroponics. Acta Hortic. 1996, 440, 205-210. Available online: https: //www.ncbi.nlm.nih.gov/pubmed/11541573 (accessed on 14 February 2019). [CrossRef] [PubMed]

21. Han, C.; Zhao, Y.; Leonard, S.W.; Traber, M. Edible coatings to improve storability and enhance nutritional value of fresh and frozen strawberries (Fragaria $\times$ ananassa) and raspberries (Rubus ideaus). Postharvest Biol. Technol. 2008, 33, 67-78. Available online: https://www.sciencedirect.com/science/article/abs/pii/S0925521404000274 (accessed on 14 February 2019). [CrossRef]

22. Rivero, R.M.; Ruiz, J.M.; Garcia, P.C.; López-Lefebre, L.R.; Sánchez, E.; Romero, L. Resistance to cold and heat stress: Accumulation of phenolic compounds in tomato and watermelon plants. Plant Sci. 2001, 160, 315-321. Available online: https://www.sciencedirect.com/science/article/pii/S0168945200003952 (accessed on 14 February 2019). [CrossRef]

23. Morand, P.; Gullo, J.L. Mineralisation des tissus vegetaux en vue du dosage de P, Ca, Mg, Na, K. Ann. Agron. 1970, 21, 229-236.

24. Fogg, D.N.; Wilkinson, N.T. The colorimetric determination of phosphorus. Analist 1958, 83, $406-414$. [CrossRef]

25. Pedrero, Z.; Madrid, Y.; Camara, C. Selenium species bioaccessibility in enriched radish (Raphanus sativus): A potential dietary source of selenium. J. Agric. Food Chem. 2006, 54, 2412-2417. Available online: https://www.ncbi.nlm.nih.gov/pubmed/16536627 (accessed on 14 February 2019). [CrossRef]

26. Hatfield, D.L.; Tsuji, P.A.; Carlson, B.A.; Gladyshev, V.N. Selenium and selenocysteine: Roles in cancer, health, and development. Trends Biochem. Sci. 2014, 39, 112-120. Available online: https://www.ncbi.nlm.nih. gov/pubmed/24485058 (accessed on 14 February 2019). [CrossRef] [PubMed]

27. Roman, M.; Jitaru, P.; Barbante, C. Selenium biochemistry and its role for human health. Metallomics 2014, 6, 25-54. Available online: https://www.ncbi.nlm.nih.gov/pubmed/24185753 (accessed on 14 February 2019). [CrossRef]

28. Malagoli, M.; Schiavon, M.; dell'Acqua, S.; Pilon-Smits, E.A.H. Effects of selenium biofortification on crop nutritional quality. Front. Plant Sci. 2015, 6, 280. Available online: https://www.ncbi.nlm.nih.gov/pubmed/ 25954299 (accessed on 14 February 2019). [CrossRef] 
29. Golubkina, N.A.; Kosheleva, O.V.; Krivenkova, L.V.; Dobrutskaya, H.G.; Nadezhkin, S.; Caruso, G. Intersexual differences in plant growth, yield, mineral composition and antioxidants of spinach (Spinacia oleracea L.) as affected by selenium form. Sci. Hortic. 2017, 225, 350-358. Available online: https://www.sciencedirect.com/ science/article/pii/S0304423817304107 (accessed on 14 February 2019). [CrossRef]

30. Hawrylak-Nowak, B. Beneficial effects of exogenous selenium in cucumber seedlings subjected to salt stress. Biol. Trace Elem. Res. 2009, 132, 259-269. Available online: https://www.ncbi.nlm.nih.gov/pubmed/19434374 (accessed on 14 February 2019). [CrossRef]

31. Pilon-Smits, E.A.H.; Quinn, C.F.; Tapken, W.; Malagoli, M.; Schiavon, M. Physiological functions of beneficial elements. Curr. Opin. Plant Biol. 2009, 12, 267-274. Available online: https://www.sciencedirect.com/science/ article/pii/S1369526609000326 (accessed on 14 February 2019). [CrossRef]

32. Hajiboland, R.; Amjad, L. Does antioxidant capacity of leaves play a role in growth response to selenium at different sulfur nutritional status? Plant Soil Environ. 2007, 53, 207-215. Available online: https: //www.agriculturejournals.cz/publicFiles/00158.pdf (accessed on 14 February 2019). [CrossRef]

33. Ferrarese, M.; Mahmoodi, M.; Quattrini, E.; Schiavi, M.; Ferrante, A. Biofortification of spinach plants applying selenium in the nutrient solution of floating system. VCRB 2012, 76, 127-136. Available online: https: //content.sciendo.com/view/journals/vcrb/76/1/article-p127.xml (accessed on 14 February 2019). [CrossRef]

34. Ávila, F.V.; Yang, Y.; Faquin, V.; Ramos, S.J.; Guilherme, L.R.G.; Thannhauser, T.W.; Li, L. Impact of selenium supply on Se-methylselenocysteine and glucosinolate accumulation in selenium-biofortified Brassica sprouts. Food Chem. 2014, 165, 578-586. Available online: https://www.ncbi.nlm.nih.gov/pubmed/25038715 (accessed on 14 February 2019). [CrossRef]

35. Institute of Medicine (US) Panel on Dietary Antioxidants and Related Compounds. Dietary Reference Intakes for Vitamin C, Vitamin E, Selenium, and Carotenoids; National Academy Press: Washington, DC, USA, 2000. Available online: https://www.ncbi.nlm.nih.gov/books/NBK225483/ (accessed on 14 February 2019).

36. Feist, L.J.; Parker, D.R. Ecotypic variation in selenium accumulation among populations of Stanleya pinnata. New Phytol. 2001, 149, 61-69. Available online: https://nph.onlinelibrary.wiley.com/doi/full/10.1046/j.14698137.2001.00004.x (accessed on 14 February 2019). [CrossRef]

37. Suarez, D.L.; Grieve, C.M.; Poss, J.A. Irrigation method affects selenium accumulation in forage Brassica species. J. Plant Nutr. 2003, 26, 191-201. Available online: https://www.tandfonline.com/doi/abs/10.1081/ PLN-120016504 (accessed on 14 February 2019). [CrossRef]

38. White, P.J.; Bowen, H.C.; Parmaguru, P.; Fritz, M.; Spracklen, W.P.; Spiby, R.E.; Meacham, M.C.; Mead, A.; Harriman, M.; Trueman, L.J.; Smith, B.M.; Thomas, B.; Broadley, M.R. Interactions between selenium and sulphur nutrition in Arabidopsis thaliana. J. Exp. Bot. 2004, 55, 1927-1937. Available online: https: //www.ncbi.nlm.nih.gov/pubmed/15258164 (accessed on 14 February 2019). [CrossRef] [PubMed]

39. Ríos, J.J.; Rosales, M.A.; Blasco, B.; Cervilha, L.; Romero, L.; Ruiz, J.M. Biofortification of Se and induction of the antioxidant capacity in lettuce plants. Sci. Hortic. 2008, 116, 248-255. Available online: https: //www.sciencedirect.com/science/article/pii/S0304423808000113 (accessed on 14 February 2019). [CrossRef]

40. Boldrin, P.F.; Faquin, V.; Ramos, S.J.; Boldrin, K.V.F.; Ávila, F.W.; Guilherme, L.R.G. Soil and foliar application of selenium in rice biofortification. J. Food Compost. Anal. 2013, 31, 238-244. Available online: https: //www.sciencedirect.com/science/article/pii/S0889157513000744 (accessed on 14 February 2019). [CrossRef]

41. Ghasemzadeh, A.; Ghasemzadeh, N. Flavonoids and phenolic acids: Role and biochemical activity in plants and human. J. Med. Plant Res. 2011, 5, 6697-6703. Available online: http://www.academicjournals.org/app/webroot/article/article1380724896_Ghasemzadeh\%20and\% 20Ghasemzadeh.pdf (accessed on 14 February 2019). [CrossRef]

42. Dixon, R.A.; Paiva, N. Stress-induced phenylpropanoid metabolism. Plant Cell 1995, 7, 1085-1097. Available online: https://www.ncbi.nlm.nih.gov/pmc/articles/PMC160915/ (accessed on 14 February 2019). [CrossRef]

43. Moglia, A.; Lanteri, S.; Comino, C.; Acquadro, A.; De Vos, R.; Beekwilder, J. Stress-induced biosynthesis of dicaffeoylquinic acids in globe artichoke. J. Agric. Food Chem. 2008, 56, 8641-8649. Available online: https://www.ncbi.nlm.nih.gov/pubmed/18710252 (accessed on 14 February 2019). [CrossRef]

44. Lee, M.J.; Lee, G.P.; Park, K.W. Effect of selenium on growth and quality in hydroponically-grown Korean Mint (Agastache rugosa). J. Korean Soc. Hortic. Sci. 2001, 42, 483-486.

45. Xu, J.; Zhu, S.; Yang, F.; Cheng, L.; Hu, Y.; Pan, G.; Hu, Q. The influence of selenium on the antioxidant activity of green tea. J. Sci. Food Agric. 2003, 83, 451-455. Available online: https://onlinelibrary.wiley.com/ doi/pdf/10.1002/jsfa.1405 (accessed on 14 February 2019). [CrossRef] 
46. Golubkina, N.; Kekina, H.; Caruso, G. Foliar biofortification of Indian mustard (Brassica juncea L.) with selenium and iodine. Plants 2018, 7, 80. [CrossRef] [PubMed]

47. Wright, M.J.; Davison, K.L. Nitrate accumulation in crops and nitrate poisoning in animals. Adv. Agron. 1964, 16, 197-247. Available online: https://www.sciencedirect.com/science/article/pii/S0065211308600255 (accessed on 14 February 2019).

48. Prasad, S.; Chetty, A.A. Nitrate-N determination in leafy vegetables: Study of the effects of cooking and reezing. Food Chem. 2008, 106, 772-780. Available online: https://www.sciencedirect.com/science/article/pii/ S0308814607005602 (accessed on 14 February 2019). [CrossRef]

49. Lei, B.; Bian, Z.; Yang, Q.; Wang, J.; Cheng, R.; Li, U.; Liu, W.; Zhang, Y.; Fang, H.; Tong, Y. The positive function of selenium supplementation on reducing nitrate accumulation in hydroponic lettuce (Lactuca sativa L.). J. Integr. Agric. 2018, 17, 837-846. Available online: https://www.sciencedirect.com/science/article/pii/ S2095311917617593 (accessed on 14 February 2019). [CrossRef]

(C) 2019 by the authors. Licensee MDPI, Basel, Switzerland. This article is an open access article distributed under the terms and conditions of the Creative Commons Attribution (CC BY) license (http://creativecommons.org/licenses/by/4.0/). 